\title{
Speed Control of DC Motors: Optimal Closed PID-Loop Model Predictive Control
}

\author{
Oluwasegun Somefun ${ }^{1, *}$, Kayode Akingbade $^{2}$, Folasade Dahunsi $^{1}$ \\ ${ }^{1}$ Department of Computer Engineering, Federal University of Technology, Akure, Ondo, Nigeria \\ ${ }^{2}$ Department of Electrical and Electronics Engineering, Federal University of Technology Akure,Ondo, Nigeria
}

Received December 8, 2019; Revised January 23, 2020; Accepted February 3, 2020

Copyright $@ 2020$ by authors, all rights reserved. Authors agree that this article remains permanently open access under the terms of the Creative Commons Attribution License 4.0 International License

\begin{abstract}
One of the lowest level control tasks, especially in robotics and other manufacturing industries, upon which other high-level controls are dependent, is the speed control of a dc motor. Usually, tuning the parameters of the proportional integral derivative (PID) controller for this task employs established conventional methods that expose the knowledge of nominal process model parameters to the control algorithm. These methods have found widespread use. Notwithstanding, a promising line of inquiry is: to search alternate possibilities of a PID being designed to automatically achieve comparable good control performance without using a formal mathematical process model approximation of the actual physical system, such as dc motor plant, in the frequency or time domain. In this paper, we propose an intelligent PID design method, "optimal closed PID-loop model predictive control", that answers this question using the characteristic settling-time (including delay-time) property of dynamic processes. The performance of this proposed method is benchmarked with popular process-model based methods. Simulation results illustrate the promise and effectiveness of the proposed tuning method, in ensuring good closed-loop performance quality for the dc motor, without using formal process models.
\end{abstract}

Keywords PID Controller, Algorithm, Adaptive Control, Optimal Control, Intelligent Control, Robust Control, DC Motor

\section{Introduction}

A generalized form of the proportional integral derivative PID structure, often referred to as the two degree-of-freedom (2DOF)-PID [1-7] fully captures the PID controller in a statefeedback plus integral form. In this paper, we introduce a 2DOF-PID controller design method, called "optimal closed PID-loop model predictive control" (OCPID-LMPC), for the intelligent speed control of a dc motor. In many applications; for instance, in mobile robotics, the speed control of a dc motor is critical to the performance of the whole system. The controller of choice for this task is mostly a PID. By conventional design methods, the PID controller heavily makes direct use of the knowledge of a nominal process model. The parameters of this formal approximation of the actual process are then used as design constants in the control algorithm [5, 8-14].

Related Works. Åström and Hägglund, in [12], place the development of design methods for the automatic tuning of the PID control algorithm as an active research in adaptive control. The tuning problem of searching for optimal PID parameters for a control task is NP-hard (nondeterministic polynomial-time) [15]. Hence, PID tuning for good control performance can be burdensome $[13,16,17]$ even for very common servomechanism applications.

It is underscored in [5, 18-21] that successful industry applications always require deep domain knowledge and understanding of process identification (plant or system modeling) $[4,11,13,14,22-28]$. Unfortunately, situations arise when these established control design methods become unreliable because of their basis on imperfect representations of the actual process dynamics. To remedy this problem, some researchers advocate for computational intensive online parameter estimations and optimizations [13,27,29].

Control practitioners [30], would prefer to avoid identification and manual tuning of PID controllers, and so alternative realistic tuning methods are welcomed. To answer this need, many model-free approaches have been proposed in literature, of note, are methods such as the unfalsified method, iterative feedback tuning, extremum-seeking method [13].

Most recent works on PID tuning in the literature such as [31-35] are devoted to employing metaheuristic optimization algorithms as new PID tuning methods. This way, different objective functions and performance constraints such as stability, bandwidth, and robustness are considered for tuning the PID. However, these methods are often carried out offline using the nominal process model, and so any serious uncertainty, as can be found in practice, in the parameters of the system can lead to instability. Again, in some cases, the optimal solution may 
never be found [27]. Therefore these methods have not found scalable use in the industry [36].

The OCPID-LMPC method, in this paper, generalizes the settling-time based method in [28]. It differs from the processmodel based design convention in the PID literature [7]. Instead, it is based on a critic 2DOF-PID structure, and the characteristic delayed settling-time property of dynamic processes. Using this approach, we show, in this paper, that the PID can be designed to automatically achieve good quality of control performance by using only the settling time of the actual dc motor plant dynamics.

Contributions. Naturally, control intelligence can be viewed as PID control. The PID control idea imitates the nature of criticism-based human cognitive actions based on the intuitive experience of error. It has been found in [37] that most machine learning algorithms in real-life applications are special cases of PID control structures.

Critic PID. As the synthesis of intelligence in automatic control algorithms continues to be a major research endeavour [9, 11, 37-39]; a future direction in controller design [9] are methods that integrate the wealth of concepts and paradigms in classical and modern controls with the science of intelligence to advance the synthesis of intelligent controllers, which will improve productivity of processes in the world.

Although, intelligent approaches that explore fuzzy logic, neural network, and proposed reinforcement learning architectures for PID design and tuning exist, these methods do not consider the idea of the PID as a critic-based cognitive structure [40]. In this paper, we show how the PID structure can be formulated in a form that more closely reflects the criticism in human learning by introducing critic weights that criticize the contributions of the three-term 2DOF-PID structure. This proposal is a future basis for fusing inference and learning by means of critic or reinforcement weights into the PID structure.

Optimal Closed PID-Loop Model Predictive Control. It is important to note that the contribution of this paper does not follow the approaches of conventional fuzzy-logic PID control techniques and direct model reference adaptive methods in the control literature. In this paper we show a nominal process model-free adaptive method for systematically optimizing the response of a process with only its characteristic timing information known by the PID control intelligence. The basis of this approach is what we have termed the common-sense ideal (or optimal) closed-loop predictive behaviour possessed by the PID controller. This optimal closed-PID loop predictive model is the intrinsic closed-loop behavioural model of the PID controller. Our approach uses a fuzzy inference of this predictive optimal model with respect to the weights of the 2DOF-PID. An augmented adaptive proportional gain update is designed to continuously adapt the three contributions in the PID, so that the process output state follows the predicted model output state.

The overarching contribution of this paper is a systematic PID design method that considers only the characteristic average settling time and delay time information, a more reliable dynamic property of a actual physical process to be controlled, in synthesizing the closed-loop belief model or behaviour of the PID controller instead of using a nominal process model

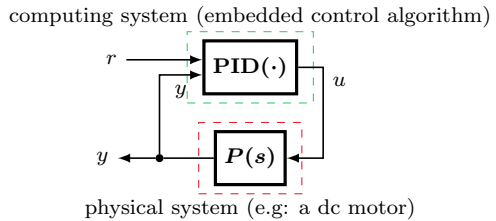

Figure 1. Overview of an embedded PID control software loop.

approximation.

The rest of this paper is organized as follows: in section 2, the critic PID and control problem is formulated; next in section 3 , the OCPID-LMPC method is developed; then in section 4, a tuning algorithm is presented; followed by section 5 , where the performance of the proposed tuning method is evaluated on a benchmark PID process as proof of concept, then applied to the speed-control of a dc motor plant; finally, section 6 concludes this paper.

\section{Problem Formulation}

In this section, the control problem is formulated by introducing critic gains for each of the three PID contributing terms to loosely reflect a reinforcement learning idea.

\subsection{Critic PID Controller}

Consider a stable linear time-invariant (LTI) and single-input single-ouput (SISO) plant with unknown dynamic structure in controllable canonical state-space form (1), which can be compactly represented as $P(s)$, as shown, in Figure 1 .

$$
\left\{\begin{array}{l}
\dot{x}_{p}=A_{p} x_{p}+B_{p} u+\Phi \\
y=C_{p} x_{p}
\end{array}\right.
$$

where $x_{p} \in \mathbb{R}^{n_{x} \times 1}$ represents $n_{x}$ states of the plant, $u \in \mathbb{R}^{1}$ is the bounded single control input to the plant, $y \in \mathbb{R}^{1}$ is the bounded single output state of the plant, $\boldsymbol{A}_{\boldsymbol{p}} \in \mathbb{R}^{n_{x} \times n_{x}}$ is a unknown stable state matrix, $\boldsymbol{B}_{\boldsymbol{p}} \in \mathbb{R}^{n_{u} \times 1}$ is the command input matrix, $\boldsymbol{C}_{\boldsymbol{p}} \in \mathbb{R}^{1 \times n_{x}}$ is the output matrix and $\boldsymbol{\Phi} \in \mathbb{R}$ is the unknown lumped uncertainty (noise, and disturbance) affecting the plant's operating conditions.

Feedback control is motivated by uncertainty. Dealing with uncertainty is a practical issue in nature and control design. Nonlinear (robust and adaptive) control techniques are used to deal with this problem in the literature. In most practical cases, this uncertainty is fuzzy and its exact magnitude cannot be measured. However, the controller must be able to operate properly in the presence of a wide range of uncertainty.

Closer to how regulation occurs in nature, the PID control idea is a computational imitation of natural robust adaptive neural feedback control intelligence. Controllers are often implemented as an embedded software in order to control the open-loop plant or process $P(s)$. To control the output state $y$ of this plant, consider the critic 2DOF PID structure in (2). In (2), the introduced terms $\lambda_{p}, \lambda_{i}, \lambda_{d} \in \mathbb{R}^{+}$are termed the "critic" weights for each of the three PID contributing terms 
$u_{p}, u_{i}, u_{d} \in \mathbb{R}$, from each of the error terms $e_{p}, e_{i}, e_{d} \in \mathbb{R}$.

$$
u=f(r, y)=\lambda_{p} u_{p}+\lambda_{i} u_{i}+\lambda_{d} u_{d}
$$

where

$$
\begin{gathered}
u_{p}=K_{p} e_{p}, \quad u_{i}=K_{i} e_{i}, \quad u_{d}=K_{d} e_{d} \\
e_{p}=(b r-y) \equiv(b r(s)-y(s)) \\
e_{i}=\int_{0}^{\tau}(r-y) d t=\int_{0}^{\tau} e d t \equiv \frac{e(s)}{s} \\
e_{d}=c \dot{r}-\dot{y} \equiv s(c r(s)-y(s))
\end{gathered}
$$

where $K_{p} \in \mathbb{R}$ is the proportional gain, $K_{i} \in \mathbb{R}$ is the integral gain, $K_{d} \in \mathbb{R}$ is the derivative gain, $u \in \mathbb{R}$ is the control action signal, $e \in \mathbb{R}$ is the error signal, $r$ is the set-point command, $y \in \mathbb{R}$ is the measured plant state or output signal.

This critic formulation (2) differs from the conventional 2DOF PID definition which assumes that the three terms equally dominate the final output decision of the PID, that is $\lambda_{i}, \lambda_{p}, \lambda_{d} \approx 1$. Also, when $\lambda_{i} \approx 0, \lambda_{p}, \lambda_{d} \neq 0$, it reduces to a form of the classical 2DOF PD structure; and when $\lambda_{d} \approx 0, \lambda_{p}, \lambda_{i} \neq 0$, it reduces to a form of the classical 2DOF PI structure. As noted in [41], these are simplistic formulations of the natural error-based feedback control principle.

The parallel critic 2DOF PID structure defined above has two inherent closed loop transfer functions available in its structure using respective proportional and derivative set-point limiters or weights $b$ and $c \in[0,1] \in \mathbb{R}^{+}[1,3,10,42-44]$. The structure reduces to the common $1 \mathrm{DOF}$ error-feedback PID structure when the set-point weights are both unity. By the definition in [6], the control law's degree of freedom (DOF) is the integervalued number of closed-loop transfer functions that are present in the controller's structure and can be adjusted independently which in this case is equal to 2 as shown in (7a). The control output, $u$ of this generalized continuous-form PID control function, has a distinct signal path to the reference set-point $r$ and the output tracked signal $y$ as illustrated in Figure 2. This kind of control structure exhibits the separation principle [10,45] that the design problems of set-point tracking and robustness plus disturbance rejection can be achieved independently and at the same time in a control law $[4,46]$.

Simplifying (2) by assuming $\lambda_{p}, \lambda_{i}, \lambda_{d}=1$ and rearranging, the result is a compact PID structure given by $(7 \mathrm{~d})$ and illustrated in Figure 2.

$$
\begin{gathered}
u=\left(K_{p} b+\frac{K_{i}}{s}+K_{d} s c\right) r- \\
\left(K_{p}+\frac{K_{i}}{s}+K_{d} s\right) y \\
u=\left(K_{p} b+K_{d} s c\right) r+\frac{K_{i}}{s} e-\left(K_{p}+K_{d} s\right) y \\
u=B(s) r(s)+A(s) e(s)-D(s) y(s) \\
u=B r+A e-D y
\end{gathered}
$$

where $B, A, D$ are variables used to simplify the loop expressions in (7b), $s$ is the complex Laplace operator (the $s$ notation will sometimes be removed from transfer functions for simplicity).

The design problem then is to appropriately control the actual $P(s)$ using the reduced PID expression (7d) without using

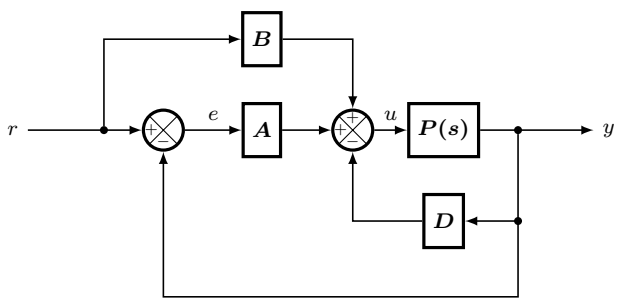

Figure 2. Internal Block diagram of the 2DOF PID control structure.



Figure 3. Signal flow graph/map (SFG) for Figure 2.

the knowledge of an identified process model to make control predictions as is required by conventional PID control design methods.

\section{Tuning Design}

In this section, the Optimal Closed PID-Loop Model Predictive Control (OCPID-LMPC) tuning method is designed for the critic PID structure defined in section 2. The goal here is to adjust the PID parameters without using a dynamic process model to represent $P(s)$.

\subsection{Closed PID-Loop}

Tuning, without any loss of generality, implies a mathematically sound approach to adjusting the main gains $K_{p}, K_{i}$ and $K_{d}$ of the PID structure in order to achieve a specified closedloop performance for $P(s)$. In other words, finding the control gains that will push or pull a non-linear system mapping to a set-point [37].

$$
C=\frac{P(s)}{1+D P(s)}
$$

To achieve this goal, the first step is to use classical methods to obtain the transfer function $T(s)$ of the overall closed loop system Figure 2. By performing a block reduction analysis (see (8), then signal-flow analysis (see Figure 3) using the Mason's Gain Formula (9), where $N$ is the number of forward paths from the input to output, $P_{i}$ is the $i_{t h}$ forward path gain, $\Delta$ is the signal flow determinant, and $\Delta_{i}$ is the co-factor of $\Delta$ along the $i_{\text {th }}$ forward path. $\Delta_{i}$ is obtained from $\Delta$ by removing the loops not touching the $i_{t h}$ forward path in Figure 3. $G(s), H(s)$, and $G(s) H(s)$ respectively represent the overall forward path gain, the overall feedback path gain, and the loop gain or transfer function of the PID loop.

$$
T(s)=\frac{y(s)}{r(s)}=\frac{G(s)}{1+G(s) H(s)}=\frac{\sum_{i=1}^{N} P_{i} \Delta_{i}}{\Delta}
$$

In this case, $N=2$. The forward path gains are: $P_{1}=A C$ and $P_{2}=B C$, with a loop gain, $L_{1}=-A C$. Therefore, 
$\Delta=1-L_{1}=1+A C, \Delta_{1}=1$ and $\Delta_{2}=1$. Applying (5), the expression (10) for the closed loop transfer function of Figure 2 is derived.

$$
T=\frac{C(A+B)}{1+A C}=\frac{(A+B) P}{1+(D+A) P}
$$

\subsection{Stability Analysis}

Theorem 3.1. The closed $2 D O F$ PID-loop transfer function $T(s)$, with the characteristic equation $1+(D+A) P(s)$, is Hurwitz stable and achieves asymptotic performance as $s \rightarrow 0$, if the PID controller (2) defined by its three main gains, $K_{p}$, $K_{i}$ and $K_{d}$, and critic weights $\lambda_{p}, \lambda_{i}$ and $\lambda_{d}$, approximates the closed-loop response behaviour $T(s)$ to a optimal second-order closed-loop model belief form $T_{m}(s)$, with the characteristic equation $s^{2}+2 \zeta \omega_{n} s+\omega_{n}^{2}$, in order to achieve asymptotic reference following of a command input trajectory $y_{m}$.

Proof. To prove Theorem 3.1, we make the following restrictive assumptions for the closed PID-loop characteristic equation $1+(D+A) P(s)$ given by the denominator of $(10)$ :

Assumption 1. Although all physical systems are nonlinear, the process $P(s)$ is designed to operate in a linear or an approximately linear and bounded input-output range. The boundedinput bounded-output (BIBO) definition of stability states that: a system is stable if every bounded input produces a bounded output.

Assumption 2. If the dynamics of $P(s)$ is BIBO stable and also affine in control according to Assumption 1. Then the unknown characteristic equation of $P(s)$ is Hurwitz (all its eigenvalues lie in the open left-half of the complex s-plane).

Assumption 3. If Assumptions 1-2 are satisfied. As $s \rightarrow 0$, then the loop gain magnitude of the closed PID-loop controlled plant will dominate the unity of its characteristic equation, that is, $|(D+A) P(s)| \gg 1$.

Remark. Following Assumption 3, we note that the intuition, according to H.S. Black's 1927 idea [44,47,48] of the negative feedback amplifier, to designing a successful negative feedback system is to make the loop gain very much larger than unity under all conditions of interest. Then the closed loop gain will not be dominated by the dynamics of the process $P(s)$.

If Assumptions 1-3 above hold, then, (10) can be approximated to (11). Where (11) which can be interpreted as the "common sense" or "closed loop predictive model" of the 2DOF PID control function (2) with respect to the output plant response $y$. This is equivalent to (12), which is a general second-order optimal transfer function response. It also can be viewed as a general interpretation of the model-following adaptive control paradigm [7] for PIDs.

$$
\begin{gathered}
\lim _{s \rightarrow 0} T \rightarrow T_{m}=\frac{A+B}{D+A}=\frac{c s^{2}+b \frac{K_{p}}{K_{d}} s+\frac{K_{i}}{K_{d}}}{s^{2}+\frac{K_{p}}{K_{d}} s+\frac{K_{i}}{K_{d}}} \\
\frac{y}{r} \approx \frac{y_{m}}{r}=\frac{c s^{2}+b 2 \zeta \omega_{n} s+\omega_{n}^{2}}{s^{2}+2 \zeta \omega_{n} s+\omega_{n}^{2}}
\end{gathered}
$$

where $\omega_{n}$ is the natural frequency, $\zeta$ is the damping factor and $y_{m}$ is the optimal output response achievable by the closed PID-loop and reference input to the closed PID-loop system.

Often, the zeros in the approximate closed loop transfer function (11) that is now the PID's model response is not desirable, so we can introduce a low pass set-point or reference input filter $F(s)$ which gives a filtered set-point $\hat{r}$ defined in (13) which transforms (11) to $\hat{T}_{m}(s)$ defined by (14). This is equivalent to setting both $b$ and $c$ to zero in (11).

$$
\begin{gathered}
F=\frac{\hat{r}}{r}=\frac{\frac{K_{i}}{K_{d}}}{c s^{2}+b \frac{K_{p}}{K_{d}} s+\frac{K_{i}}{K_{d}}}=\frac{1}{\frac{c}{\omega_{n}^{2}} s^{2}+b \frac{2 \zeta}{\omega_{n}} s+1} \\
\hat{T}_{m}=\frac{\omega_{n}^{2}}{s^{2}+2 \zeta \omega_{n} s+\omega_{n}^{2}}
\end{gathered}
$$

The expected closed PID-loop eigenvalues (15) is obtained from the optimal characteristic denominator equation of (12), with $\zeta$ taking a value of 0.707 , for a optimal or near optimal [49] and robust [50] output response.

$$
\begin{aligned}
s_{1,2}=-\zeta \omega_{n} & \pm j \omega_{n} \sqrt{1-\zeta^{2}}=-\kappa \omega_{d} \pm j \omega_{d} \\
\kappa & =\zeta \frac{\omega_{n}}{\omega_{d}}=\frac{\zeta}{\sqrt{1-\zeta^{2}}}
\end{aligned}
$$

where $\omega_{d}$ is the damped natural frequency of oscillation or imaginary part of the conjugate complex poles, $\kappa$ is the exponential decay factor, and the product $\kappa \omega_{d}=\zeta \omega_{n}$ is the exponential decay frequency or real part of the poles of the closed loop PID model response.

This completes the proof.

\subsection{Setting $K_{d}$ and $K_{i}$}

Comparing (11) and (12), the result is the following nominal identities (17) and (18) for the respective gain and time-constant of the derivative and integral parts of the PID.

$$
\begin{array}{cc}
K_{d}=f_{d}\left(K_{p}, \omega_{n}, \zeta\right)=K_{p} \frac{1}{2 \zeta \omega_{n}}, \quad T_{d}=\frac{1}{2 \zeta \omega_{n}} \\
K_{i}=f_{i}\left(K_{p}, \omega_{n}, \zeta\right)=K_{p} \frac{\omega_{n}}{2 \zeta}, \quad T_{i}=\frac{2 \zeta}{\omega_{n}}
\end{array}
$$

The set PID parameters (17) and (18) agrees with the result of [51] obtained by minimizing the quadratic cost function

$$
\begin{gathered}
V=\int_{0}^{\infty}\left[(r-y(t))^{2}+\kappa_{p}^{2}(u(t)-u(\infty))^{2}\right] \\
=\int_{0}^{\infty}\left[e(t)^{2}+\Delta u(t)^{2}\right] \\
u(\infty)=\frac{r}{\kappa_{p}} .
\end{gathered}
$$

where $\kappa_{p}$ is the steady state process gain.

\subsection{Closed PID-Loop Model Response}

The expressions (17) and (18) set $K_{i}$ and $K_{d}$ but depends on $K_{p}$ and $w_{n}$. To progress towards a solution, in this section, we analyze the output state motion of closed loop predictive model (11) of the 2DOF-PID algorithm. 


\subsubsection{Normalized Model Output Response}

The analytic time-domain response of the model can be obtained. A partial fraction analysis (PFA) of (14) leads to (23). Next, by taking the inverse laplace transformation (ILT), we obtain expressions for the output response (21) and derivative (25) of the closed PID-loop model transfer function $T_{m}(s)$ to a unit step input, $r(s)=\frac{1}{s}$. The result is (24) and (28) respectively.

$$
\begin{gathered}
y_{m}(s)=\frac{c s^{2}+b 2 \zeta \omega_{n} s+\omega_{n}^{2}}{s\left(s^{2}+2 \zeta \omega_{n} s+\omega_{n}^{2}\right)} \\
=\frac{k_{A}}{s}+\frac{k_{B} s+k_{C}}{s^{2}+2 \zeta \omega_{n} s+\omega_{n}^{2}}
\end{gathered}
$$

where

$$
\begin{gathered}
k_{A}=1, k_{B}=c-1, k_{C}=(b-1) 2 \zeta \omega_{n} \\
y_{m}(t)=1+e^{-\zeta \omega_{n} t}\left[\begin{array}{l}
(c-1) \cos \left(\omega_{d} t\right)+ \\
(2 b-c-1) \zeta \frac{\omega_{n}}{\omega_{d}} \sin \left(\omega_{d} t\right)
\end{array}\right]
\end{gathered}
$$

Also,

where

$$
\begin{aligned}
s y_{m}(s) & =T_{m}(s)=\frac{c s^{2}+b 2 \zeta \omega_{n} s+\omega_{n}^{2}}{s^{2}+2 \zeta \omega_{n} s+\omega_{n}^{2}} \\
& =c+\frac{k_{1} s+k_{2}}{s^{2}+2 \zeta \omega_{n} s+\omega_{n}^{2}}
\end{aligned}
$$

$$
\begin{gathered}
k_{1}=(b-c) 2 \zeta \omega_{n}, k_{2}=\omega_{n}^{2}(1-c) \\
\dot{y}_{m}(t)=c+e^{-\zeta \omega_{n} t}\left[\begin{array}{l}
(b-c) 2 \zeta \omega_{n} \cos \left(\omega_{d} t\right)+ \\
\omega_{n}^{2}\left(\frac{(1-c)}{\omega_{d}}-\frac{(b-c) 2 \zeta^{2}}{\omega_{d}}\right) \\
\zeta \frac{\omega_{n}}{\omega_{d}} \sin \left(\omega_{d} t\right)
\end{array}\right]
\end{gathered}
$$

If we let the normalized time at $\omega_{n}=1$, be $x=\zeta \omega_{n} t$, then the normalized model output response (24) and normalized model output response derivative (28) can be expressed compactly as (30) and (31) respectively.

$$
\begin{gathered}
\text { Let } \kappa=\zeta \frac{\omega_{n}}{\omega_{d}}=\frac{\zeta}{\sqrt{1-\zeta^{2}}} \\
\omega_{d} t=\frac{x}{\kappa} \\
y_{m}(x)=1+e^{-x}\left[\begin{array}{l}
(c-1) \cos \left(\frac{x}{\kappa}\right)+ \\
(2 b-c-1) \kappa \sin \left(\frac{x}{\kappa}\right)
\end{array}\right] \\
\dot{y}_{m}(x)=c+e^{-x}\left[\begin{array}{l}
(b-c) 2 \zeta \cos \left(\frac{x}{\kappa}\right)+ \\
\left(\frac{(1-c)}{\zeta}-(b-c) 2 \zeta\right) \\
\kappa \sin \left(\frac{x}{\kappa}\right)
\end{array}\right]
\end{gathered}
$$

\subsubsection{State-space Response Analysis}

In observable canonical state-space form (32), the closedPID loop model response $T_{m}(s)$ can be re-expressed as (33).

$$
\begin{gathered}
\dot{\boldsymbol{x}}=\boldsymbol{A} \boldsymbol{x}+\boldsymbol{B} \boldsymbol{r} \\
\boldsymbol{y}_{\boldsymbol{m}}=\boldsymbol{C} \boldsymbol{x}+\boldsymbol{D} \boldsymbol{r} \\
\boldsymbol{x}=\left[\begin{array}{l}
x_{1} \\
x_{2}
\end{array}\right], \dot{\boldsymbol{x}}=\left[\begin{array}{l}
\dot{x}_{1} \\
\dot{x}_{2}
\end{array}\right]
\end{gathered}
$$

where,

$$
\begin{gathered}
\boldsymbol{A}=\left[\begin{array}{lll}
0 & -\omega_{n}^{2} 1 & -2 \zeta \omega_{n}
\end{array}\right] \boldsymbol{B}=\left[\begin{array}{c}
\omega_{n}^{2}(1-c) \\
2 \zeta \omega_{n}(b-c)
\end{array}\right] \\
\boldsymbol{C}=\left[\begin{array}{ll}
0 & 1
\end{array}\right] \boldsymbol{D}=[c]
\end{gathered}
$$

Further analyzing this state-space solution by assuming a constant or slowly varying set-point command signal $r$ leads to the derivation of the following interesting relationships (34)-(40).

$$
\begin{gathered}
y_{m}=x_{2}+c r \therefore x_{2}=y_{m}-c r \\
\therefore e_{d, m}=-\dot{x}_{2} \text { and } \therefore \dot{y}_{m}=\dot{x}_{2} \\
\dot{x}_{1}=\omega_{n}^{2}\left(-x_{2}+(1-c) r\right) \\
\therefore e_{m}=r-y_{m}=\frac{\dot{x}_{1}}{\omega_{n}^{2}} \quad \therefore e_{i, m}=\frac{e_{m}}{s}=\frac{x_{1}}{\omega_{n}^{2}} \\
\dot{x}_{2}=x_{1}+2 \zeta \omega_{n}\left(-x_{2}+(b-c) r\right) \\
\therefore e_{p, m}=b r-y_{m}=\frac{\dot{x}_{2}-x_{1}}{2 \zeta \omega_{n}}
\end{gathered}
$$

leading to

$$
u_{m}=K_{p} e_{p, m}+K_{i} e_{i, m}+K_{d} e_{d, m}
$$

and

$$
e_{u, m}=u(\infty)-u_{m}
$$

The industrial form of the PID favours the I-PD structural form ( $b=0, c=0$ ) equivalent to a integral plus state feedback controller, meanwhile a more general form favours the PI-D form ( $b=1, c=0)$. With respect to these common PID forms, using the derived relationships from the state-space solution (33), we graphically observe the model output and error response that a PID controlled closed loop plant should follow. This is illustrated in the two example normalized plots in Figure 4 and Figure 5.

It is observed that the response of the closed 2DOF-PID loop model is affected by the choice of $b$ and $c$. Changing values of $b$ and $c$ lead to variations in the normalized peak times and settling times of $T_{m}(s)$ the closed PID-loop model response which affects the PID's model input-output surface. The plot of the input-output surface of the PID model, as illustrated in Figure $4 \mathrm{~h}$ and Figure 5h, reveals a convex shaped mapping curve.

In the next section, in order to set the model natural frequency $w_{n}$, we analyze the normalized plots of the model output response and its first derivative. The first undershoot area of the model output response is considered to obtain its approximate normalized settling time. For the normalized peak time, the first point at which the derivative of the model output response is zero is considered. The result is Table 1 and 2.

\subsection{Setting $\omega_{n}$ by fuzzy inference of the closed PID-loop response}

The fact that the output response of a BIBO stable physical system does not instantaneously settle to a steady state value, due to the inherent input energy dissipation of physical systems [52], leads to an intuitive choice of the settling time plus delay time of the open-loop physical process in setting the model closed-loop natural frequency $\omega_{n}$. 
Table 1. Normalized Peak Time: Closed PID-Loop Model's Fuzzy Membership Function Centers, $\bar{c}^{1}$

\begin{tabular}{|c|c|c|c|c|c|c|c|c|c|c|c|}
\hline \multirow{2}{*}{$c$} & \multicolumn{11}{|c|}{$b$} \\
\hline & 0 & 0.1 & 0.2 & 0.3 & 0.4 & 0.5 & 0.6 & 0.7 & 0.8 & 0.9 & 1 \\
\hline 0 & 4.43 & 4.27 & 4.1 & 3.86 & 3.6 & 3.32 & 3 & 2.78 & 2.55 & 2.36 & 2.2 \\
\hline 0.1 & 4.29 & 4.15 & 3.99 & 3.79 & 3.54 & 3.26 & 2.96 & 2.68 & 2.44 & 2.23 & 2.07 \\
\hline 0.2 & 4.41 & 4.3 & 4.15 & 3.97 & 3.73 & 3.44 & 3.11 & 2.78 & 2.49 & 2.26 & 2.07 \\
\hline 0.3 & 4.54 & 4.44 & 4.32 & 4.15 & 3.94 & 3.65 & 3.3 & 2.92 & 2.57 & 2.28 & 2.07 \\
\hline 0.4 & 4.66 & 4.58 & 4.48 & 4.34 & 4.15 & 3.89 & 3.54 & 3.11 & 2.68 & 2.33 & 2.07 \\
\hline 0.5 & 4.76 & 4.7 & 4.63 & 4.52 & 4.37 & 4.15 & 3.83 & 3.37 & 2.85 & 2.39 & 2.07 \\
\hline 0.6 & 4.87 & 4.82 & 4.77 & 4.69 & 4.58 & 4.41 & 4.15 & 3.73 & 3.11 & 2.49 & 2.07 \\
\hline 0.7 & 4.96 & 4.93 & 4.89 & 4.84 & 4.77 & 4.65 & 4.48 & 4.15 & 3.54 & 2.68 & 2.07 \\
\hline 0.8 & 5.04 & 5.02 & 5.0 & 4.97 & 4.93 & 4.87 & 4.76 & 4.58 & 4.15 & 3.11 & 2.07 \\
\hline 0.9 & 5.12 & 5.11 & 5.1 & 5.09 & 5.07 & 5.04 & 5 & 4.93 & 4.77 & 4.15 & 2.07 \\
\hline 1 & 5.5 & 5.5 & 5.5 & 5.5 & 5.5 & 5.5 & 5.5 & 5.5 & 5.5 & 5.5 & 5.5 \\
\hline
\end{tabular}

Table 2. Normalized Settling Time: Closed PID-Loop Model's Fuzzy Membership Function Centers, $\bar{c}^{1}$

\begin{tabular}{|c|c|c|c|c|c|c|c|c|c|c|c|}
\hline \multirow{2}{*}{$c$} & \multicolumn{10}{|c|}{$b$} \\
\cline { 2 - 15 } & 0 & 0.1 & 0.2 & 0.3 & 0.4 & 0.5 & 0.6 & 0.7 & 0.8 & 0.9 & 1 \\
\hline 0 & 9.98 & 9.81 & 9.61 & 9.39 & 9.13 & 8.85 & 8.58 & 8.31 & 8.09 & 7.89 & 7.74 \\
\hline 0.1 & 9.38 & 9.25 & 9.09 & 8.88 & 8.64 & 8.36 & 8.06 & 7.78 & 7.54 & 7.33 & 7.17 \\
\hline 0.2 & 9.51 & 9.4 & 9.25 & 9.07 & 8.83 & 8.54 & 8.21 & 7.88 & 7.59 & 7.36 & 7.17 \\
\hline 0.3 & 9.64 & 9.54 & 9.42 & 9.25 & 9.04 & 8.75 & 8.4 & 8.02 & 7.67 & 7.38 & 7.17 \\
\hline 0.4 & 9.76 & 9.68 & 9.58 & 9.44 & 9.25 & 8.99 & 8.64 & 8.21 & 7.78 & 7.43 & 7.17 \\
\hline 0.5 & 9.86 & 9.81 & 9.73 & 9.62 & 9.47 & 9.25 & 8.93 & 8.47 & 7.95 & 7.49 & 7.17 \\
\hline 0.6 & 9.96 & 9.92 & 9.86 & 9.79 & 9.68 & 9.51 & 9.25 & 8.83 & 8.21 & 7.59 & 7.17 \\
\hline 0.7 & 10.06 & 10.03 & 9.99 & 9.94 & 9.86 & 9.75 & 9.58 & 9.25 & 8.64 & 7.78 & 7.17 \\
\hline 0.8 & 10.14 & 10.12 & 10.1 & 10.07 & 10.03 & 9.96 & 9.86 & 9.66 & 9.25 & 8.21 & 7.17 \\
\hline 0.9 & 10.22 & 10.21 & 10.2 & 10.18 & 10.17 & 10.14 & 10.1 & 10.03 & 9.86 & 9.25 & 7.17 \\
\hline 1 & 11.07 & 11.07 & 11.07 & 11.07 & 11.07 & 11.07 & 11.07 & 11.07 & 11.07 & 11.07 & 11.07 \\
\hline
\end{tabular}

${ }^{1} \bar{d}=2.22$ if $b=0$ or $b=1$, otherwise $\bar{d}=2.08$

From the classical time-domain relation $\omega_{n}=\frac{x}{\zeta t}$, the desired closed loop natural frequency $\omega_{n}$ at the normalized settling time $x=x_{s}$ with respect to the operating settling time $t_{s}$ of the actual open-loop process can be calculated. Alternatively, the desired $\omega_{n}$ may also be calculated using the normalized peak time $x=x_{p k}$ value, with respect to the operating open-loop peak time value $t_{p k}$.

$$
\omega_{n}=f_{\omega_{n}}\left(\zeta, x_{s}, t_{s}\right)=\frac{x_{s}}{\zeta t_{s}} \quad \text { or } \quad \omega_{n}=\frac{x_{p k}}{t_{p k} \sqrt{1-\zeta^{2}}}
$$

To compute $\omega_{n}$ using (42), our approach is to encode the fuzzy behaviour of the optimal response of the closed PID-loop model with changing $b$ and $c$ as a fuzzy inference system (FIS), defined by (44), and synthesized as a fuzzy basis function (FBF) [8] defined in (45). This method is used to automatically compute the appropriate $x_{s}$ or $x_{p k}$ with respect to the inputs $b$ and $c$ respectively. The design choice for this FIS is outlined in Table 3, while the input-output nonlinear mapping surface of the FIS is shown in Figure 6.

Table 3. Type-I FIS Design Choice

\begin{tabular}{|c|c|c|}
\hline FIS Type & \multicolumn{2}{|c|}{ Takagi-Sugeno-Kang (TSK) } \\
\hline Input(s) & \multicolumn{2}{|c|}{$b$ and $c \therefore p=2$} \\
\hline Antecedent Fuzzifier & \multicolumn{2}{|c|}{ Singletons } \\
\hline Universe & \multicolumn{2}{|c|}{$0-1$} \\
\hline Output(s) & $x_{p k}$ & $x_{t s}$ \\
\hline Consequent Fuzzifier & Singleton & Non-Singleton \\
\cline { 2 - 3 } Universe & $2-6$ & $0-20$ \\
\cline { 2 - 3 } T-norm & Product & Product \\
\cline { 2 - 3 } T-conorm & Nil & Max \\
\hline MF (Parameterized) & closed $n$-logistic sigmoids, see (45) \\
\hline MF Parameters & \multicolumn{2}{|c|}{ Pre-specified, see Table 1 and 2 } \\
\hline Number of Rules & \multicolumn{2}{|c|}{$M=121$ for each output } \\
\hline
\end{tabular}

Definition 3.1. For input $\boldsymbol{x}=\left[\begin{array}{ll}b & c\end{array}\right]^{T}$, the Type-1 FIS that maps to $\boldsymbol{y}=\left[\begin{array}{ll}x_{s} & x_{p k}\end{array}\right]^{T}$ is represented as the fuzzy basis 




(a)

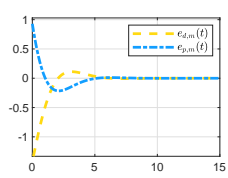

(d)



(b)

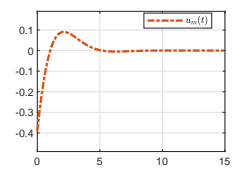

(e)

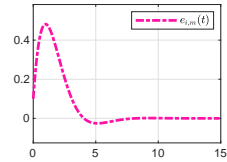

(c)

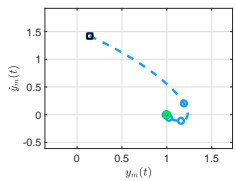

(f)

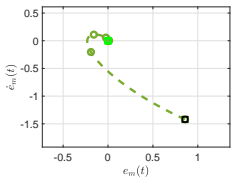

(g)

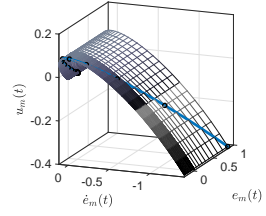

(h)
Figure 4. Closed PID-loop model's state space response analysis, when $b=1$, $c=0$; (4a) output $y_{m}$ and error $e_{m},(4 \mathrm{~b})$ output rate $\dot{y}_{m}$ and error rate $\dot{e}_{m}$ (4c) integral error $e_{i, m}$, (4d) proportional $e_{p, m}$ and derivative $e_{d, m}$ errors, (4e) control output $u_{m}$ responses, (4f) output phase plot, (4g) error phase plot, and (4h) control output surface map.

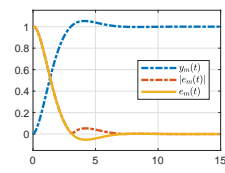

(a)

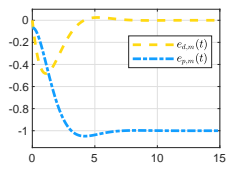

(d)

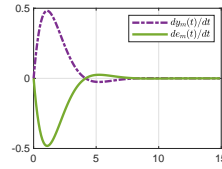

(b)

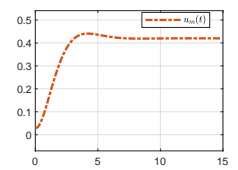

(e)

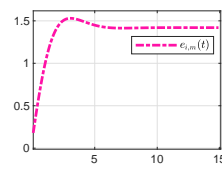

(c)

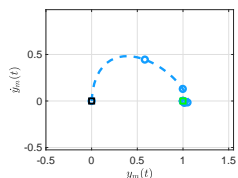

(f)

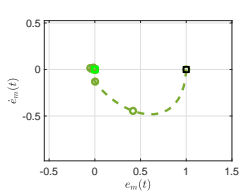

(g)

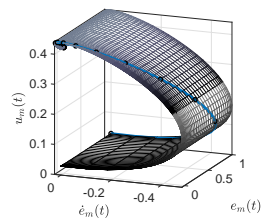

(h)
Figure 5. Closed PID-loop model's state space response analysis, when $b=0$, $c=0$; (5a) output $y_{m}$ and error $e_{m},(5 \mathrm{~b})$ output rate $\dot{y}_{m}$ and error rate $\dot{e}_{m}$ (5c) integral error $e_{i, m},(5 \mathrm{~d})$ proportional $e_{p, m}$ and derivative $e_{d, m}$ errors, (5e) control output $u_{m}$ responses, (5f) output phase plot, (5g) error phase plot, and $(5 \mathrm{~h})$ control output surface map.

function expansion:

$$
\begin{gathered}
\boldsymbol{y}(\boldsymbol{x})=\sum_{l=1}^{M} c_{0}^{l} \phi_{j}^{l}(\boldsymbol{x}) \\
\phi_{j}^{l}(\boldsymbol{x})=\frac{\prod_{i=1}^{p} \mu_{F_{i}}^{l}\left(x_{i}\right)}{\sum_{l=1}^{M} \prod_{i=1}^{p} \mu_{F_{i}}^{l}\left(x_{i}\right)} \\
\mu_{F_{i}}^{l}\left(x_{i}\right)=\left\{\begin{array}{l}
\mu_{L}(x)=\mathrm{nlsig}^{-}\left(x ; \bar{c}_{L}, \bar{d}_{L}\right) ; x<\frac{\bar{c}_{L}+\bar{c}_{R}}{2} \\
\mu_{R}(x)=\operatorname{nlsig}^{+}\left(x ; \bar{c}_{R}, \bar{d}_{R}\right) ; x \geqslant \frac{\bar{c}_{L}+\bar{c}_{R}}{2}
\end{array}\right.
\end{gathered}
$$

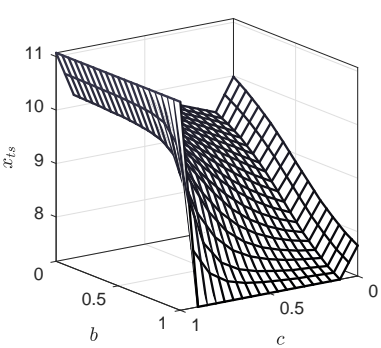

(a)

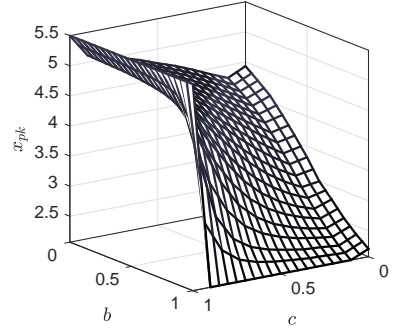

(b)
Figure 6. FIS Output surface defined by (44) for the closed PID-loop model.

For $n l$ sig $^{-}$, the following constrains hold $x_{\min }^{-}=\bar{c}_{L}-\bar{d}_{L}$, $x_{\max }^{-}=\bar{c}_{L}, \bar{c}_{L}>x_{\min }$ and $x_{\min }^{+}=\bar{c}_{R}, x_{\max }^{+}=\bar{c}_{R}+\bar{d}_{R}$, $\bar{c}_{R}<x_{\max }$ for $n l s i g^{+}$. Also, $y_{\max }=1, y_{\min }=0$.

Definition 3.2. The $n$-logistic sigmoid function, where $\delta \in$ $\mathbb{R}^{n \times 1}$ and $\kappa_{x}, \kappa_{y} \in \mathbb{R}^{(n+1) \times 1}$ with $\lambda=6$ as a standard default value is defined as:

$$
\begin{aligned}
y & =\operatorname{nlsig}^{ \pm}\left(x ; x_{\min }, x_{\max }, n, \lambda\right) \\
& =\kappa_{y, 1}+\sum_{i=1}^{n} \frac{\kappa_{y, i+1}-\kappa_{y, i}}{1+e^{ \pm \alpha\left(x-\delta_{i}\right)}}
\end{aligned}
$$

The following holds:

$$
\begin{gathered}
\left\{\begin{array}{l}
\lim _{x \rightarrow x_{\max }} \mathrm{nlsig}^{-}(x)=y_{\max } \\
\lim _{x \rightarrow x_{\min }} \mathrm{nlsig}^{-}(x)=y_{\min } \\
\lim _{x \rightarrow x_{\min }} \mathrm{nlsig}^{+}(x)=y_{\max } \\
\lim _{x \rightarrow x_{\max }} \mathrm{nlsig}^{+}(x)=y_{\min }
\end{array}\right. \\
\Delta_{x}=\frac{x_{\max }-x_{\min }}{n}, \quad \Delta_{y}=\frac{y_{\max }-y_{\min }}{n} \\
\alpha=\lambda \frac{2}{\kappa_{x, i+1}-\kappa_{x, i}}=\lambda \frac{2}{\kappa_{x, 2}-\kappa_{x, 1}} \\
\delta_{i}=\frac{\kappa_{x, i+1}+\kappa_{x, i}}{2}, \quad i=1, \ldots, n \\
\kappa_{x, i+1}=\kappa_{x, i}+\Delta_{x}, \quad \kappa_{y, i+1}=\kappa_{y, i}+\Delta_{y}
\end{gathered}
$$

where $\kappa_{x}=\left[\kappa_{x, i}, \ldots, \kappa_{x, i+1}\right], \kappa_{x, 1}=x_{\min }, \kappa_{x, n+1}=x_{\max }$ and $\kappa_{y}=\left[\kappa_{y, i}, \ldots, \kappa_{y, i+1}\right], \kappa_{y, 1}=y_{\min }, \kappa_{y, n+1}=y_{\max }$.

\subsection{Setting $K_{p}$}

The remaining PID parameter to set is the proportional gain $K_{p}$. Practically, the power limitations (noise and saturation) of the hardware actuator, which parses the controller's output to effect a process output, limits how high $K_{p}$ can be set. From (17) and (18), it is clear that $K_{p}$ is the PID's global gain parameter upon which the two other gain parameters are dependent. The inquiry then is: how to determine a stabilizing real-valued $K_{p}$ that will effectively regulate $P(s)$ and constrain the actuator's energy within limits without resulting to the use of a process model? In this paper, we propose an hybrid solution.

First, our approach is to set $y_{m}$ as the input to the PID and tune $K_{p}$ as an adaptive function of the resulting error $y_{m}-y$. In standard controllable state form, the closed PID-loop model 
is

$$
\begin{gathered}
\dot{\boldsymbol{x}}_{\boldsymbol{m}}=\boldsymbol{A}_{\boldsymbol{m}} \boldsymbol{x}_{\boldsymbol{m}}+\boldsymbol{B}_{\boldsymbol{m}} r \\
y=\boldsymbol{C}_{\boldsymbol{m}} \boldsymbol{x}_{\boldsymbol{m}}+\boldsymbol{D}_{\boldsymbol{m}} r \\
\boldsymbol{A}_{\boldsymbol{m}}=\left[\begin{array}{cc}
0 & 1 \\
-\omega_{n}^{2} & -2 \zeta \omega_{n}
\end{array}\right] \boldsymbol{B}_{\boldsymbol{m}}=\left[\begin{array}{l}
0 \\
1
\end{array}\right] \\
\boldsymbol{C}_{\boldsymbol{m}}=\left[\omega_{n}^{2}(1-c)\right. \\
\left.2 \zeta \omega_{n}(b-c)\right] \boldsymbol{D}_{\boldsymbol{m}}=[c]
\end{gathered}
$$

If we assume the control law is

$$
u=u_{p}=K_{p} e_{p}=K_{p}\left(b x_{m}-x_{p}\right)
$$

Assuming an attenuated effect of plant uncertainty, the unknown plant state dynamics (1) is $\dot{\boldsymbol{x}}_{\boldsymbol{p}}=\boldsymbol{A} \boldsymbol{x}_{\boldsymbol{p}}+\boldsymbol{B} u$. The tracking error is $e=x_{m}-x$ and its derivative is $\dot{e}=\dot{x}_{m}-\dot{x}_{p}$. For perfect closed-loop model matching or following, $x_{p}=x_{m}$ and $x_{m}=r$, then we have

$$
\dot{x}_{p}=\left(\boldsymbol{A}-\boldsymbol{B} K_{p}\right) x_{p}+\boldsymbol{B} K_{p} b x_{m}
$$

Comparing (55) with (52), we obtain the following identities

$$
\begin{gathered}
\boldsymbol{A}_{\boldsymbol{m}}=\boldsymbol{A}-\boldsymbol{B} K_{p}(\infty) \quad \boldsymbol{B}_{\boldsymbol{m}}=\boldsymbol{B} K_{p}(\infty) \\
\therefore \boldsymbol{A}=\boldsymbol{A}_{\boldsymbol{m}}+\frac{\boldsymbol{B}_{\boldsymbol{m}}}{b}
\end{gathered}
$$

The tracking error becomes

$$
\dot{e}=\boldsymbol{A}_{\boldsymbol{m}} e+\boldsymbol{B}\left(K_{p}(\infty)-K_{p}\right) e_{p}
$$

As a candidate lyapunov function $V$, we choose a quadratic function of the error and $K_{p}$,

$$
V\left(e, K_{p}\right)=\gamma \frac{e^{2}}{2}+\boldsymbol{B} \frac{\left(K_{p}(\infty)-K_{p}\right)^{2}}{2}
$$

Then its derivative $\dot{V}$ is

$$
\begin{gathered}
\dot{V}=\gamma e \dot{e}+\boldsymbol{B}\left(K_{p}(\infty)-K_{p}\right) \dot{K}_{p} \\
\dot{V}=\gamma \boldsymbol{A}_{\boldsymbol{m}} e^{2}+\boldsymbol{B}\left(K_{p}(\infty)-K_{p}\right)\left(\gamma e e_{p}+\dot{K}_{p}\right)
\end{gathered}
$$

The parameter update law can be selected as

$$
\begin{gathered}
\dot{K}_{p}=-\gamma e e_{p}, \quad \gamma=0.001 \\
\text { if } b=1, \quad \dot{K}_{p}=-\gamma e^{2} \\
K_{p}=K_{p}-\dot{K}_{p}
\end{gathered}
$$

Using Barbalat's lemma it is easy to see that $e$ asymptotically converges to zero as time goes to infinity. This implies that the controlled plant output $y$ tends to the closed-PID loop model output $y_{m}$. An initial value for $K_{p}$ can be taken as 0.01 , where $\gamma$ is also a very small positive constant.

However, there is always a form of delay, even if negligible, in most processes. When this delay gets larger, the solution (62) obtained from the approach above cannot alone guarantee the stability of the closed-loop system. To solve this problem, we apply an augmented approach.

To set $K_{p}$ within appropriate bounds, our approach is to use a nonlinear sum of error, $e$ and the control action $u$ using the forward form of the newly formulated n-logistic sigmoid function $n l s i g^{-}$in Definition (3.2), with, $y_{\max }=x_{\max }=k_{\text {lim }}$, and $y_{\min }=x_{\min }=0$. We wish to constrain $K_{p}$ to a value between 0 and $k_{\text {lim }}$. However, the maximum possible positive stabilizing value $k_{\text {lim }}$ is unknown. To obtain an approximate value for $k_{\text {lim }}$, we analyse a PID-controlled normalized first-order plus dead time model with increasing values of normalized delay time $L$. The obtained data values of $L$ and $k_{\lim }$ that leads to a stabilizing $K_{p}$ value is fitted to a normalized rational function (68) multiplied by the ratio of delayed settling-time to the settling-time without delay. The overall solution (64) is expressed below.

$$
K_{p}=f_{p}\left(e, u, k_{\lim }\right)=n l s i g^{-}\left(k_{0} ; 0, k_{\lim }, n_{p}, \lambda_{k}\right)
$$

where:

$$
\begin{gathered}
k_{0}=n l s i g^{-}\left(e(t) ;-k_{\lim }, k_{\lim }, n_{e}, \lambda_{k}\right) \\
+n l s i g^{-}\left(u(t) ;-k_{\lim }, k_{\lim }, n_{u}, \lambda_{k}\right) . \\
n_{p}=16, \quad n_{e}=n_{u}=1, \quad \lambda_{k}=0.1 \\
k_{\lim }=\alpha \kappa_{g}\left(\frac{L+t_{s}}{t_{s}}\right), \quad k_{\lim }, \alpha \in \mathbb{R}^{+}
\end{gathered}
$$

By default, $\alpha=1$

$$
\begin{gathered}
\kappa_{g}=\frac{p_{1} \bar{L}^{2}+p_{2} \bar{L}+p_{3}}{\bar{L}^{2}+q_{1} \bar{L}+q_{2}} \\
p_{1}=0.05132 \quad p_{2}=0.2041 \quad p_{3}=0.1214 \\
q_{1}=1.538 \quad p_{2}=0.5864 \\
\bar{L}=\frac{L-5.936}{8.771}
\end{gathered}
$$

when $t>L$

$$
K_{p}=K_{p}-\dot{K}_{p}
$$

The sigmoidal limiting function (64) is formulated in such a way that it estimates and constrains the maximum value of $K_{p}$ with respect to a free variable hyper-parameter $\alpha$ and the presence of a delay $L$ in the settling time.

Remark. The realization of Theorem 3.1 for different physical systems depends on the value of the constrained global PID gain parameter $K_{p}$ defined with (64). However, provided the Assumptions (1)-(3) are satisfied, the output step response of a PID controlled system should asymptotically converge towards the stable response of the closed PID-loop model response.

\section{SAD Tuning Algorithm}

Based on the optimal closed PID-loop model predictive control method proposed in section 3, in this section, we present an automatic tuning algorithm named "SAD".

For a periodic triggered control framework, where $T \leq 0.01$ seconds is the sampling time, the SAD algorithm is outlined in Algorithm 1.

The derivative filter time-constant $T_{f}$ is usually used to make the measured error derivative causal or to attenuate the noise associated with it.

\section{Simulation Results and Discussion}

Simulation results of the proposed method, benchmarked against other selected methods in literature will be presented and discussed in this section. 


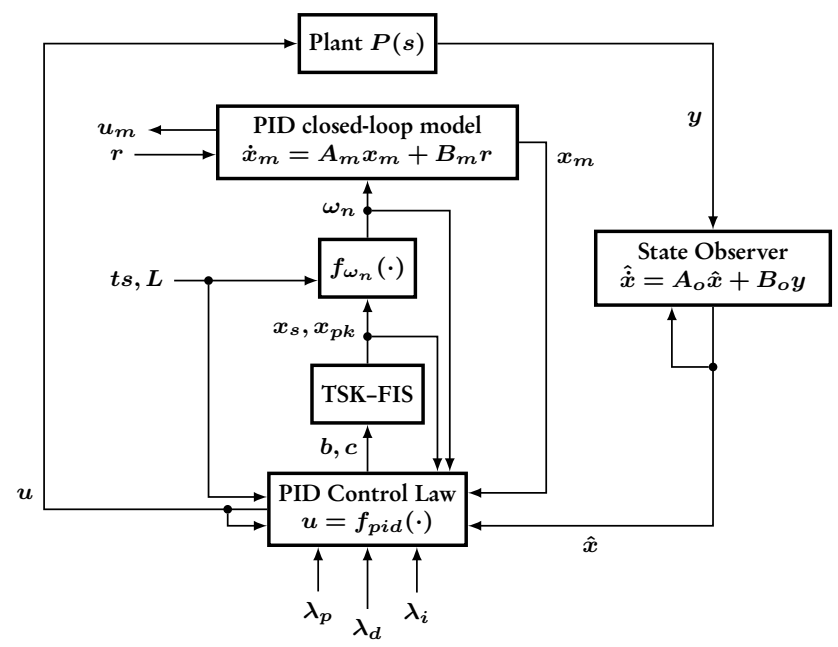

Figure 7. Overall Architecture of the Optimal Closed PID-Loop Model Predictive Control Method (OCPID-LMPC).

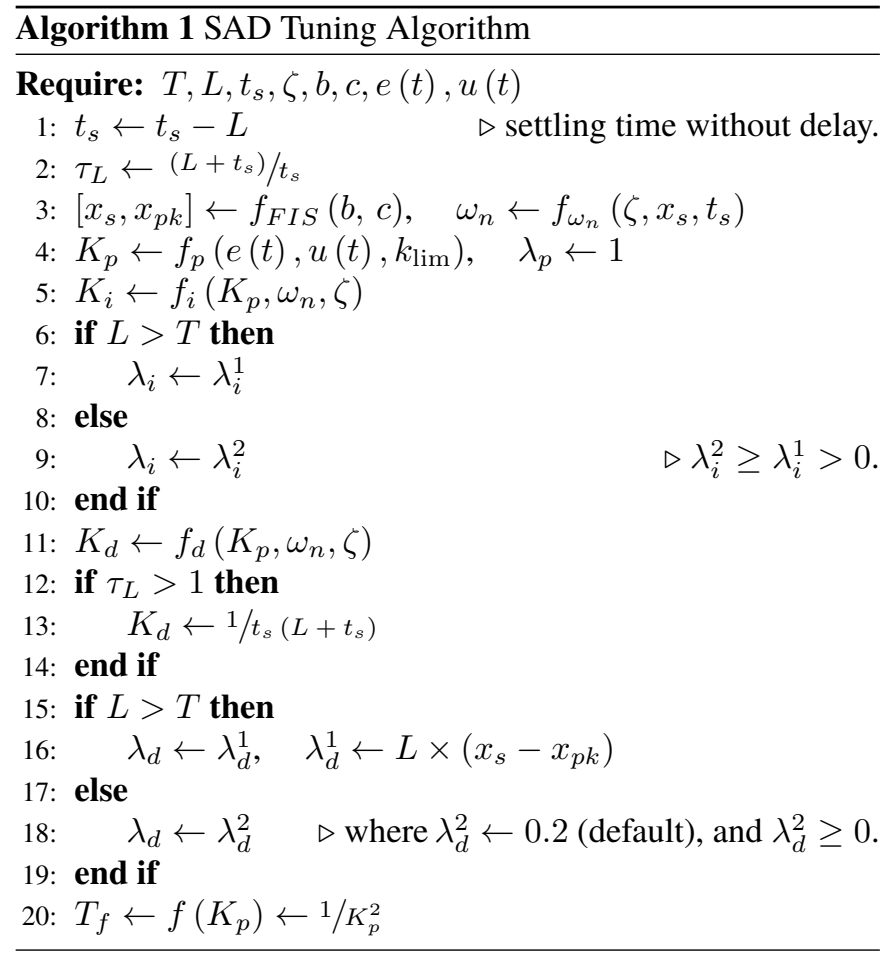

Table 4. Tuning Rules ${ }^{1}$.

\begin{tabular}{|c|c|c|c|}
\hline & $K_{p}$ & $K_{i}$ & $K_{d}$ \\
\hline CHR Chiens, Hrones and Reswick $(0 \%)^{1}$ & $0.6 \frac{\tau}{\kappa_{p} L}$ & $0.6 \frac{\kappa_{p}}{L}$ & $0.3 \frac{\tau}{\kappa_{p}}$ \\
\hline SAD Somefun, Akingbade and Dahunsi & $f_{p}(\cdot)$ & $f_{i}(\cdot)$ & $f_{d}(\cdot)$ \\
\hline
\end{tabular}

${ }^{1} 0 \%$ set-point regulation. where $\tau$ is process time-constant and $\kappa_{p}$ is steadystate gain of the process.

We have developed a method for optimizing the step response of a closed-loop system consisting of a 2DOF PID controller ( $b=1$ and $c=0$ ) and the unknown dynamics of a LTI SISO plant. Compared to the Z-N methods, instead of using a stepresponse experiment to obtain a first-order plus dead time plant model estimate, we use this experiment to estimate the delayed settling time of the plant as its characteristic linear dynamic behaviour. The effect of this is that we restrict the use of identified process models as representations of the true process for simulation purposes and not for the control design.

As standard performance measures, the quadratic integral performance error indices in form of $J_{i s e}$ and $J_{i s u}$ are good measures of set-point regulation. Where: $e(t)$ is the error due a unit step change in the set-point command signal, $u(t)$ is the control input from the controller, $u(\infty)$ is the optimal steadystate control input corresponding to the desired output. Also, an instinctive requirement for modern PID tuning methods is to ensure specified closed loop performance in terms of the maximum overshoot and settling time [2, 3, 18, 28]. Therefore, the controller tuning performance is also assessed using the settling time and percentage maximum overshoot indices of the closed-loop with respect to a unit-step input.

$$
J_{i s e}=\int_{0}^{\infty} e(t)^{2}, \quad J_{i s u}=\int_{0}^{\infty}(u(\infty)-u(t))^{2}
$$

\subsection{First Order Plus Dead Time Model}

$$
P_{1}(s)=\frac{\kappa_{p}}{\tau s+1} e^{-s L}
$$

Consider a representative first-order plus dead time (FOPDT) process model for a dc motor plant, where $\kappa_{p}=1, \tau=1, L=$ 2 . The controllability ratio of this plant is 0.67 . This implies a lag dominated (difficult to control) plant.

Applying the proposed SAD algorithm in comparison with the CHR method, JJ-ST method and the AMIGOS method; Figure 9 shows the normalized process response to a unit-step command, and Table 5 shows the steady-state controller parameters and performance.

It is deduced from the performance indices in Tables 5 that all four PID tuning methods achieve set-point regulation successfully. The AMIGO Response is smooth, no overshoot but most sluggish. Using the CHR method, the output response has the highest percentage maximum overshoot, while for the JJ-ST method, a more oscillatory response is observed. Compared to 
Table 5. Controller Parameters and Performance Measures ${ }^{1}$ for the Plant $P_{1}=\frac{1}{(s+1)} e^{-2 s}$.

\begin{tabular}{|c|c|c|c|c|c|c|c|}
\hline & $K_{p}$ & $K_{i}$ & $K_{d}$ & $J_{i s e}$ & $J_{i s u}$ & $t_{s}$ & $M_{o}$ \\
\hline \hline $\mathrm{SAD}$ & 0.172 & 0.1651 & 0.004 & 3.51 & 1.98 & 10.08 & 1.91 \\
\hline $\mathrm{JJ}_{-S T}$ & 0.6 & 0.3 & 0 & 2.37 & 1.05 & 12.5 & 7.81 \\
\hline $\mathrm{CHR}$ & 0.3 & 0.3 & 0.3 & 3.20 & 0.455 & 13.84 & 11.2 \\
\hline $\mathrm{AMIGO}^{2}$ & 0.21 & 0.175 & 0 & 3.64 & 2.08 & 15.88 & 0 \\
\hline
\end{tabular}

${ }^{1} t_{s}^{o}=10$ seconds and $t_{s}$ is the open and closed loop settling time (delay inclusive) in seconds respectively. $M_{o}$ is the percentage maximum overshoot.

2 JJ-ST (Jantzen and Jakobsen's Settling Time method). See [28].

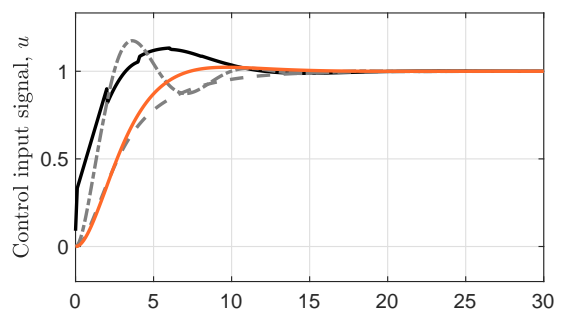

(a)

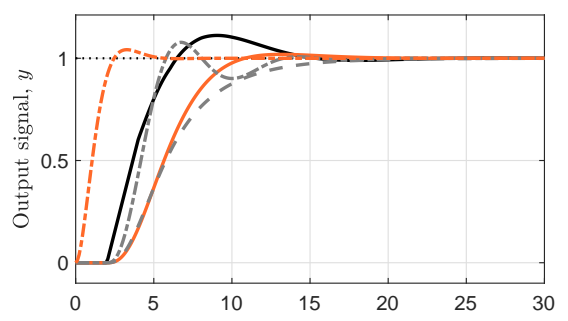

(b)

Figure 8. Responses to a unit step command for $P_{1}(s)$ : Fig. (9a.) Control signal output, Fig. (9b.) Controlled output response. SAD (solid red; $\alpha=1$., $\lambda_{i}=1.25, \lambda_{d}=11.08$ ), optimal closed PID-loop model response (dashed red), JJ-ST (dash-dotted gray), CHR (solid black), AMIGO (dashed gray).

these methods, the overall best performance is obtained by the OCPID-LMPC method through the SAD algorithm which provides the best balance between the closed-loop settling time and maximum overshoot of the output response. It, therefore, can be inferred from the above results that the OCPID-LMPC method is very capable of giving good control performance even in the presence of dead-time for this class of systems, compared to the other methods that use the knowledge of a formal process model representation of the actual plant.

\subsection{Speed Control of a DC Motor}

Next we consider a more realistic representation (oscillatory plant model) of an actual permanent magnent dc-motor plant with specifications shown in Table 6.

where:

$$
P_{2}(s)=\frac{b_{0}}{s^{2}+a_{1} s+a_{2}}
$$

Table 6. Geared Permanent Magnet (PM) DC Motor $\left(P_{2}(s)\right)$ Specifications for a Differential Drive Mobile Robot [53].

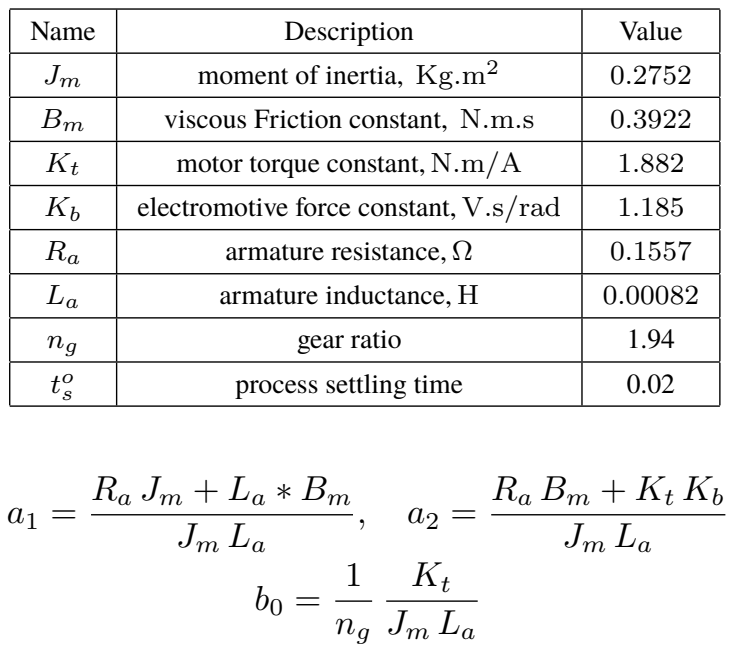

The settling time of the process output (speed) is 0.02 seconds. A sensor noise of $\pm 20 \%$ and a state noise of $\pm 1 \%$ modelled as a gaussian process is assumed for a unit-step response command. Also, a unit step disturbance was introduced at 0.2 seconds. Figure 9 illustrates the performance of the OCPID-LMPC method and varying PID gains, with respect to the set-point regulation of the dc motor's process output (speed). The resultant performance indices is $J_{\text {ise }}=0.032$.

It can be concluded from the performance measures in this section that the OCPID-LMPC method in relation to the speed control of the dc motor plant gives a robust closed loop system in the presence of bounded uncertainties acting on the measured output process.

\subsection{Discussions}

Many sophisticated estimation techniques exist for process or system identification [54]. In itself process identification helps control designers understand the limits and the input-output behaviour of the process. The proposed method, however, uses a very simplistic form of low-level process identification that can be quite easily carried out based on input-output data to estimate the process gain, delay time and settling time of the process, without formulating a process model. The use of process models is used in its primary application to simulate an approximate behaviour of the actual plant and test the performance of the tuned PID controller [55]. 


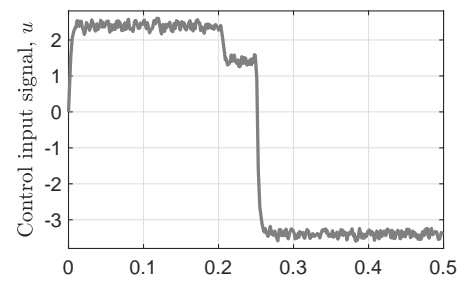

(a)

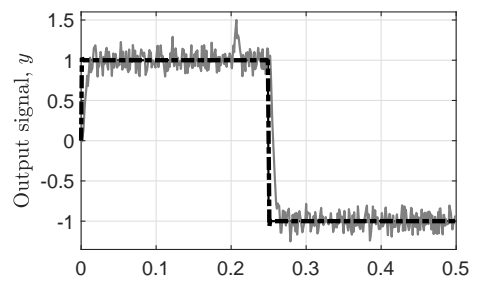

(b)



(c)

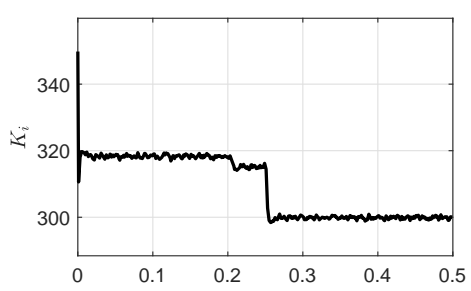

(d)

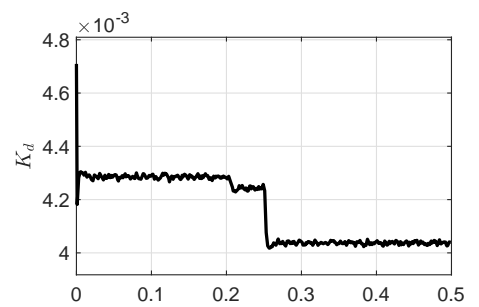

(e)

Figure 9. Responses to a unit step command for $P_{2}(s)$ : Fig. (9a.) Control signal output, Fig. (9b.) Controlled output response SAD (solid gray; $\alpha=1.33$, $\lambda_{i}=1.1, \lambda_{d}=0.67$ ), optimal closed PID-loop model response (dashed black), Fig. (9c.) Proportional Gain, Fig. (9d.) Integral Gain, Fig. (9e.) Derivative Gain.

Although PID design is regarded by many as an established area, according to $[18,56-59]$, engineering practice is persistently demanding for PID control design methods that simultaneously guarantee the metrics of maximum overshoot and settling time. Researchers [17,60-62] agree that a satisfactory controller can be a PID if its parameter gain space is well tuned or exploited. For all of the test cases provided above, the output response of the process follows the optimal closed-PID loop output response with respect to the correct estimations of the final settling time and time-delay of the open loop process.

For control tasks involving a process variable like the speed or position of a dc motor, regulating such around their operating regions can become less complex and more automatic. Good or near optimal control gains can be computed automatically without the use of uncertain exaggerated process models, hence the time and effort taken to compute optimal control actions is reduced significantly. Further, it is straightforward to see that this proposed OCPID-LMPC method has better and comparable control performance when properly applied. Therefore, this method has promising use in place of the popular model-based PID tuning methods, as a first try for tuning PIDs in dc motor applications.

\section{Conclusions}

In this paper, we first reformulated the PID structure to include critic gains which penalize each of its three terms. Next the designed OCPID-LMPC adaptive tuning method was proposed. The OCPID-LMPC method is a flexibile framework as different automatic algorithms can be designed based on it. The resulting tuning algorithm in this paper, $\mathrm{SAD}$, is fast and quite easy to implement as a software library, so that industry engineers and non-expert users can flexibly employ PIDs to achieve good quality of control performance with efficient expenditure of control energy compared to other established PID tuning methods. As proof of concept, simulation results show that this process model-free PID tuning method can approximate the optimal solution for a specific class of physical processes. For performance validation, the method was demonstrated on some benchmark systems and compared with some key PID tuning methods in the literature. From performance results, the proposed method outperforms: the CHR method in minimizing the quadratic cost function; and the convex-concave optimization in minimizing the integral absolute error. Hence we conclude that this systematic approach to PID design (tuning) has potential for applicability to many PID controllable plants even when noisy and dead-time dominated, with a promising industrial application being the robust position and speed control of electric motor drives and in motion control.

\section{REFERENCES}

[1] K. Sladká, J. Czebe, and R. Wagnerová, "The 2DOF controller implementation in control system with single-chip computer," in 2018 19th International Carpathian Control Conference (ICCC), May 2018, pp. 228-234.

[2] X. Wang, X. Yan, D. Li, and L. Sun, "An Approach for Setting Parameters for Two-Degree-of-Freedom PID Controllers," Algorithms, vol. 11, no. 4, p. 48, Apr. 2018.

[3] M. Viteckova and A. Vitecek, "2DOF Controller Tuning," International Journal of Engineering Research in Africa, vol. 18, pp. 57-64, 2015. 
[4] A. Forrai, Embedded Control System Design: A Model Based Approach. Heidelberg ; New York: Springer, 2013, oCLC: ocn811007710.

[5] S. W. Sung, J. Lee, and L. In-Beum Lee, Process Identification and PID Control. John Wiley \& Sons, Ltd, 2009.

[6] M. Araki and H. Taguchi, "Two-degree-of-freedom PID controllers," International Journal of Control, Automation, and Systems, vol. 1, no. 4, pp. 401-411, 2003.

[7] K. J. Åström and T. Hägglund, PID Controllers - Theory, Design, and Tuning, 2nd ed. ISA, 1995.

[8] J. M. Mendel, Uncertain Rule-Based Fuzzy Systems: Introduction and New Directions, 2nd ed. Springer International Publishing, 2017.

[9] L. Li, N. Zheng, and F. Wang, "On the Crossroad of Artificial Intelligence: A Revisit to Alan Turing and Norbert Wiener," IEEE Transactions on Cybernetics, vol. 49, no. 10 , pp. 3618-3626, Oct. 2019.

[10] K. J. Åström and T. Hägglund, "Design methods: PID Control," The Control Handbook: Control System Fundamentals, Second Edition, pp. 9-70-9-90, 2017.

[11] S. Li and J. Wang, "Research on Engineering Tuning Methods of PID Controller Parameters and Its Application," in Intelligent Computing Methodologies, ser. Lecture Notes in Computer Science, D.-S. Huang, K. Han, and A. Hussain, Eds. Springer International Publishing, 2016, pp. 563-570.

[12] W. S. Levine, The Control Systems Handbook: Control System Advanced Methods, Second Edition (Electrical Engineering Handbook), 2nd ed., ser. Electrical Engineering Handbooks. CRC Press, 2011.

[13] N. J. Killingsworth and M. Krstic, "PID tuning using extremum seeking: Online, model-free performance optimization," IEEE Control Systems Magazine, vol. 26, no. 1, pp. 70-79, Feb. 2006.

[14] A. Visioli, Practical PID Control. Springer Science \& Business Media, Nov. 2006.

[15] L. Koszaka, R. Rudek, and I. Pozniak-Koszalka, “An Idea of Using Reinforcement Learning in Adaptive Control Systems," in International Conference on Networking, International Conference on Systems and International Conference on Mobile Communications and Learning Technologies (ICNICONSMCL'06), Apr. 2006, pp. 190-190.

[16] T. Roux-Oliveira, L. R. Costa, A. V. Pino, P. Paz, T. RouxOliveira, L. R. Costa, A. V. Pino, and P. Paz, "Extremum Seeking-based Adaptive PID Control applied to Neuromuscular Electrical Stimulation," Anais da Academia Brasileira de Ciências, vol. 91, Jan. 2019.
[17] C. Grimholt and S. Skogestad, "Optimal PI and PID control of first-order plus delay processes and evaluation of the original and improved SIMC rules," Journal of Process Control, vol. 70, pp. 36-46, 2018.

[18] Š. Bucz and A. Kozáková, "Advanced Methods of PID Controller Tuning for Specified Performance," PID Control for Industrial Processes, Sep. 2018.

[19] T. Samad, "A Survey on Industry Impact and Challenges Thereof [Technical Activities]," IEEE Control Systems Magazine, vol. 37, no. 1, pp. 17-18, Feb. 2017.

[20] T. Samad and A. Annaswamy, "New Edition of CSS's "The Impact of Control Technology" Report [Publication Activities]," IEEE Control Systems Magazine, vol. 33, no. 2, pp. 21-21, Apr. 2013.

[21] V. Bobál, J. Böhm, J. Fessl, and J. Machácek, Digital SelfTuning Controllers: Algorithms, Implementation and Applications, ser. Advanced Textbooks in Control and Signal Processing. London: Springer-Verlag, 2005.

[22] Y. Bai and Z. S. Roth, Classical and Modern Controls with Microcontrollers: Design, Implementation and Applications, ser. Advances in Industrial Control. Springer International Publishing, 2019.

[23] K. M. Lynch, N. Marchuk, and M. L. Elwin, "PID Feedback Control," in Embedded Computing and Mechatronics with the PIC32. Elsevier, 2016, pp. 375-385.

[24] M. Li, J. Wang, and D. Li, "Performance robustness comparison of two PID tuning methods," in Proceedings of the 29th Chinese Control Conference, Jul. 2010, pp. 36013605.

[25] A. O'Dwyer, Handbook of PI and PID Controller Tuning Rules. Imperial College Press, 2009.

[26] V. Aggarwal and U. O'Reilly, "A Self-Tuning Analog Proportional-Integral-Derivative (PID) Controller," in First NASA/ESA Conference on Adaptive Hardware and Systems (AHS'06), Jun. 2006, pp. 12-19.

[27] A. A. Dastjerdi, N. Saikumar, and S. H. HosseinNia, "Tuning guidelines for fractional order PID controllers: Rules of thumb," Mechatronics, vol. 56, pp. 26-36, Dec. 2018.

[28] J. Jantzen and C. Jakobsen, "Turning PID controller tuning into a simple consideration of settling time," in 2016 European Control Conference (ECC), Jun. 2016, pp. 370375.

[29] G. Ellis, Ed., Control System Design Guide. Boston: Butterworth-Heinemann, Jan. 2012.

[30] K. M. Moudgalya, Digital Control. Wiley Online Library, 2007.

[31] S. Ekinci and B. Hekımoğlu, "Improved Kidney-Inspired Algorithm Approach for Tuning of PID Controller in AVR System," IEEE Access, vol. 7, pp. 39 935-39947, 2019. 
[32] B. Hekimoğlu, "Optimal Tuning of Fractional Order PID Controller for DC Motor Speed Control via Chaotic Atom Search Optimization Algorithm," IEEE Access, vol. 7, pp. 38 100-38 114, 2019.

[33] R. K. Mandava and P. R. Vundavilli, “An optimal PID controller for a biped robot walking on flat terrain using MCIWO algorithms," Evolutionary Intelligence, vol. 12, no. 1, pp. 33-48, Mar. 2019.

[34] A. Almabrok, M. Psarakis, and A. Dounis, "Fast Tuning of the PID Controller in An HVAC System Using the Big Bang-Big Crunch Algorithm and FPGA Technology," Algorithms, vol. 11, no. 10, p. 146, Oct. 2018.

[35] A. Taeib and A. Chaari, "Tuning optimal PID controller," International Journal of Modelling, Identification and Control, vol. 23, no. 2, pp. 140-147, Jan. 2015.

[36] M. Elwin, K. M. Lynch, and N. Marchuk, Embedded Computing and Mechatronics with the PIC32 Microcontroller, 1st ed. Newnes is an imprint of Elsevier, 2015.

[37] B. Recht, "A Tour of Reinforcement Learning: The View from Continuous Control," arXiv:1806.09460 [cs, math, stat], Jun. 2018.

[38] T. RayChaudhuri, L. G. C. Hamey, and R. D. Bell, "From conventional control to autonomous intelligent methods," IEEE Control Systems Magazine, vol. 16, no. 5, pp. 78-84, Oct. 1996.

[39] P. J. Antsaklis, K. M. Passino, and S. J. Wang, "Towards intelligent autonomous control systems: Architecture and fundamental issues," Journal of Intelligent and Robotic Systems, vol. 1, no. 4, pp. 315-342, Dec. 1989.

[40] A. Marino and F. Neri, "PID Tuning with Neural Networks," in Intelligent Information and Database Systems. Springer, Cham, Apr. 2019, pp. 476-487.

[41] J. Han, "From PID to Active Disturbance Rejection Control," IEEE Transactions on Industrial Electronics, vol. 56, no. 3, pp. 900-906, Mar. 2009.

[42] B. G. Abdelaty, A. H. Ahmed, and A. N. Ouda, "Fixed Set Point Weighting 2DOF PID Controller for Control Processes," Engineering Mathematics, vol. 2, no. 1, p. 21, Jul. 2018 .

[43] M. Vagia, "PID Controller Design Approaches-Theory, Tuning and Application to Frontier Areas," 2012.

[44] K. J. Astrom and T. Hagglund, Advanced PID Control. ISA Instrumentation, Systems and Automation Society, 2006.

[45] W. Wang, "The New Design Strategy on PID Controllers," in PID Controller Design Approaches - Theory, Tuning and Application to Frontier Areas, M. Vagia, Ed. InTech, Mar. 2012.

[46] L. Keviczky and C. Bányász, Two-Degree-of-Freedom Control Systems. Elsevier, 2015.
[47] J. Mbihi, Analog Automation and Digital Feedback Control Techniques, 1st ed., ser. Systems and Industrial Engineering. Wiley-ISTE, 2018.

[48] M. Thompson, Intuitive Analog Circuit Design, Second Edition, 2nd ed. Newnes, 2013.

[49] C. Yi, "Correcting the minimum ITAE standard forms of zero-displaceemnt-error systems," Journal of Zhejiang University (Natural Science), vol. 23, no. 4, pp. 550-559, 1989.

[50] I. J. Nagrath and M. Gopal, Control Systems Engineering. New Age International, 2006.

[51] K. Peter and R. Isermann, "Parameter-Adaptive Control Based on Continuous-Time Process Models," IFAC Proceedings Volumes, vol. 23, no. 8, Part 2, pp. 443-448, Aug. 1990.

[52] A. Tewari, Modern Control Design: With MATLAB and SIMULINK. Wiley, Apr. 2002.

[53] A. A. Mahfouz, A. A. Aly, and F. A. Salem, "Mechatronics Design of a Mobile Robot System," International Journal of Intelligent Systems and Applications(IJISA), vol. 5 , no. 3, p. 23.

[54] J. Böling, Identification for Control with Application to IllConditioned Systems. AAbo Akademi University Turku, Finland, 2001.

[55] K. J. Åström and T. Hägglund, "Benchmark Systems for PID Control," IFAC Proceedings Volumes, vol. 33, no. 4, pp. 165-166, Apr. 2000.

[56] Y. Ashida, S. Wakitani, and T. Yamamoto, "Design of a Performance-Adaptive 1-Parameter Tuning PID Controller," Proceedings of International Conference on Artificial Life and Robotics, vol. 23, pp. 326-329, Feb. 2018.

[57] D. P. Atherton, "Setting the Parameters of Proportional-Integral-Derivative Controllers," Measurement and Control, vol. 48, no. 9, pp. 273-277, Nov. 2015.

[58] K. Ogata and Y. Yang, Modern Control Engineering. Prentice hall India, 2002, vol. 4.

[59] K. J. Åström, "Control System Design Lecture notes for ME155A,” 2002.

[60] K. Soltesz and A. Cervin, "When is PID a good choice?" IFAC-PapersOnLine, vol. 51, no. 4, pp. 250-255, Jan. 2018.

[61] J. E. Normey-Rico, Control of Dead-Time Processes, ser. Advanced Textbooks in Control and Signal Processing. London: Springer-Verlag, 2007.

[62] G. J. Silva, A. Datta, and S. P. Bhattacharyya, PID Controllers for Time-Delay Systems, ser. Control Engineering. Birkhäuser Basel, 2005. 